\title{
Élaboration, fiabilité et utilisation d'un outil de mesure de l'environnement alimentaire dans les supermarchés de quatre quartiers de Montréal (Canada)
}

\author{
Élise Jalbert-Arsenault, Dt. P. (1); Éric Robitaille, Ph. D. (1,2); Marie-Claude Paquette, Ph. D. (1,2)
}

Cet article a fait l'objet d'une évaluation par les pairs.

Diffuser cet article sur Twitter

\section{Résumé}

Introduction : L'environnement alimentaire constitue un domaine propice si l'on veut influencer les habitudes alimentaires de la population. Cette étude visait à élaborer un outil de mesure de l'environnement alimentaire dans les commerces et à caractériser l'environnement alimentaire dans un secteur de Montréal (Canada) possédant un niveau de revenu de faible à moyen.

Méthodologie : Nous avons développé un outil, la Mesure de l'environnement alimentaire du consommateur dans les supermarchés (MEAC-S) et nous l'avons mis à l'essai pour en confirmer la fiabilité. Nous avons utilisé la MEAC-S pour évaluer l'environnement alimentaire de consommation de 17 supermarchés dans quatre quartiers de Montréal. Nous avons mesuré la longueur d'étalage, la diversité, le prix, le nombre de présentoirs et la position en magasin des fruits et légumes (FL) et des produits alimentaires ultratransformés (PAUT). Nous avons également évalué la qualité des FL frais. La taille du magasin a été estimée en utilisant la longueur totale d'étalage mesurée pour toutes les catégories d'aliments. Nous avons effectué des corrélations de Spearman entre ces indicateurs de l'environnement alimentaire.

Résultats : Les analyses de fiabilité ont donné des résultats satisfaisants pour la plupart des indicateurs. La caractérisation de l'environnement alimentaire a révélé une grande variabilité de la longueur d'étalage, de la diversité et du prix des FL entre les supermarchés ainsi qu'une mise en valeur disproportionnée des PAUT. Le nombre de présentoirs de PAUT hors de leur emplacement principal de vente variait de 7 à 26 et ces produits occupaient de 8 à 33 positions stratégiques en magasin, alors que le nombre de présentoirs hors de l'emplacement principal de vente de FL frais était supérieur à 1 dans seulement 2 des 17 magasins évalués et qu'ils occupaient un maximum de 2 emplacements stratégiques par supermarché. Le prix des PAUT était inversement associé à leur proéminence $(p<0,005)$ et à leur promotion $(p<0,003)$. La taille du magasin était associée au nombre de présentoirs et au positionnement stratégique des PAUT $(p<0,001)$ mais pas à celui des FL, et elle était inversement associée au prix des boissons gazeuses $(p<0,003)$.

Conclusion : Cette étude illustre la variabilité de l'environnement alimentaire entre les supermarchés et souligne l'importance de mesurer les caractéristiques en magasin pour brosser un tableau juste de l'environnement alimentaire du consommateur.

Mots-clés : nutrition, environnement alimentaire, environnement alimentaire de consommation, fruits et légumes, transformation des aliments, marketing alimentaire, obésité, produits alimentaires ultra-transformés

\section{Introduction}

Plus de la moitié des adultes canadiens sont en surpoids $(36,8 \%)$ ou obèses $(25,1 \%)^{1}$. Cela constitue un important fardeau social et financier pour le pays : jusqu'à $12 \%$ des dépenses totales en santé au Canada seraient attribuables à l'obésité ${ }^{2}$. $\mathrm{Au}$ Québec seulement, le coût annuel de l'excès de poids a été estimé à 3 milliards
Points saillants

- L’outil MEAC-S a été conçu pour évaluer et surveiller l'environnement alimentaire de consommation à Montréal (Canada) et a démontré une fiabilité interévaluateur robuste.

- La disponibilité et le prix des fruits et légumes varient considérablement selon les supermarchés.

- Les produits alimentaires ultratransformés, à la différence des fruits et légumes, font l'objet d'une promotion considérable et disproportionnée dans les supermarchés, promotion qui augmente avec la taille du magasin.

- En matière d'environnement alimentaire communautaire, la dichotomisation des commerces d'alimentation comme étant fournisseurs d'aliments sains ou non sains ne reflète pas avec justesse l'environnement alimentaire auquel les consommateurs sont exposés.

de dollars ${ }^{3}$. Parallèlement, les comportements alimentaires, considérés comme l'un des principaux déterminants du poids et comme un facteur de risque modifiable pour le développement de nombreuses maladies non transmissibles ${ }^{4}$, ne sont pas optimaux au Québec. La consommation moyenne de fruits et de légumes (FL) dans la population adulte est inférieure à cinq portions par jour 5 . Une analyse récente des données pour le Québec de l'Enquête sur la santé dans les collectivités canadiennes, Cycle 2.2, Nutrition (2004) a également révélé que les produits alimentaires ultratransformés (PAUT) représentent près de la 
moitié des calories consommées $(47 \%)^{6}$ dans la province.

Il a été démontré que l'environnement alimentaire influence les choix et les habitudes alimentaires ${ }^{7}$. L'environnement alimentaire communautaire (accessibilité à différents types de magasins alimentaires) et de consommation (ce qui est disponible dans les magasins d'alimentation) ont été associés à la consommation de $\mathrm{FL}^{8-13}$, à la qualité du régime alimentaire ${ }^{8,11,14-18}$ et au poids $^{19-25}$. Au Canada, l'environnement alimentaire actuel offre des aliments de haute densité énergétique et des PAUT peu coûteux, facilement disponibles et faisant l'objet d'une promotion très importante ${ }^{26}$. Avec un environnement alimentaire aussi déséquilibré, l'éducation en matière de nutrition n'est probablement pas suffisante pour améliorer les habitudes alimentaires de la population ${ }^{27,28}$. Il est essentiel de modifier l'environnement alimentaire de façon à ce que le choix sain constitue le choix facile si l'on veut commencer à freiner l'augmentation de la prévalence de l’obésité.

L'environnement alimentaire au Canada et au Québec n'est pas bien documenté à l'heure actuelle. Cette pénurie de données entrave la capacité à orienter, à élaborer et à mettre en œuvre des interventions et des politiques susceptibles de favoriser une alimentation saine ${ }^{29}$. Dans un article récent, Minaker et ses collègues ${ }^{30}$ soulignent en particulier le manque de recherche sur l'environnement alimentaire de consommation au Canada, un seul article ayant utilisé des mesures de l'environnement alimentaire de consommation pour étudier l'association entre l'environnement alimentaire et les résultats sur la santé. Alors que la proximité et la disponibilité de magasins dans un quartier ont été liées à la qualité de l'alimentation, les études ont fait état de résultats incohérents ${ }^{31}$, ce qui suggère que l'accessibilité physique pourrait ne pas suffire à expliquer les habitudes alimentaires. La disponibilité et le coût des aliments dans ces magasins pourraient contribuer à l'association entre l'accès aux magasins d'alimentation et le choix du lieu d'approvisionnement, les comportements alimentaires et les résultats sur la santée ${ }^{32,33}$.

Plus de 30 outils de mesure de l'environnement alimentaire de consommation ont été identifiés ${ }^{34}$. Les deux outils les plus fréquemment utilisés sont l'Enquête sur les mesures de l'environnement nutritionnel dans les magasins (Nutrition Environment Measures Survey in Stores, NEMS-S) ${ }^{35}$ et le USDA Thrifty Food Plan ${ }^{36}$. Ces outils permettent de décrire la disponibilité et le prix de produits alimentaires variés. Le NEMS-S évalue également la qualité des fruits et légumes frais. Ni l'un ni l'autre, comme la plupart des autres outils de mesure ${ }^{34}$, ne décrivent la mise en valeur des aliments ou la proéminence des catégories d'aliments dans les magasins d'alimentation, malgré l'influence de ces facteurs sur les décisions d'achats alimentaires ${ }^{37-40}$.

Dans ce contexte, les objectifs de notre étude étaient : (1) de concevoir un outil de mesure pour les magasins d'alimentation qui intègre les composantes de l'environnement alimentaire de consommation telles que définies dans le Modèle des environnements alimentaires communautaires élaboré par Glanz et ses collègues, en plus de la mise en valeur et du positionnement des aliments ${ }^{7}$, et (2) de caractériser l'environnement alimentaire de consommation dans un secteur (4 quartiers) de Montréal (Canada) possédant un niveau de revenu de faible à moyen.

\section{Méthodologie}

\section{Élaboration de l'outil}

L'outil Mesure de l'environnement alimentaire du consommateur dans les supermarchés (MEAC-S) a été élaboré pour évaluer l'environnement alimentaire de consommation dans les supermarchés.

\section{Catégories d'aliments}

La MEAC-S comprend deux catégories d'aliments : ceux dont la consommation est documentée comme insuffisante (catégorie des FL) et ceux dont la consommation est documentée comme trop importante (catégorie des PAUT) selon les recommandations du Guide alimentaire canadien $^{5,6}$. La catégorie des FL comprend les FL frais, congelés, en conserve et prêts à consommer. La catégorie des PAUT, définis comme des produits alimentaires créés à partir d'ingrédients industriels et contenant peu ou pas d'aliments entiers ${ }^{41}$, comprend les croustilles, les boissons gazeuses, les plats congelés et les confiseries. Ces aliments ont été choisis parce qu'ils représentaient $11 \%$ des ventes totales dans les supermarchés au Québec en 2013-2014 ${ }^{42}$.

Les essais pilotes ont révélé que les confiseries étaient disponibles dans de multiples endroits dans les commerces et partageaient souvent l'espace d'étalage avec d'autres produits alimentaires. Ce positionnement des confiseries a empêché d'effectuer une évaluation fiable de la diversité et de la longueur d'étalage pour ces produits. De ce fait, seul le positionnement des confiseries dans des positions stratégiques en magasin a été évalué.

\section{Principaux indicateurs}

La MEAC-S permet d'évaluer la disponibilité, le prix, la mise en valeur et la promotion des deux catégories d'aliments dans les supermarchés. Les indicateurs inclus dans l'outil sont énumérés et définis ci-dessous.

1. La disponibilité des aliments a été mesurée en utilisant trois indicateurs : la diversité de produits dans chaque catégorie d'aliments, la longueur d'étalage qu'ils occupent dans le supermarché et la qualité des fruits et légumes frais.

La diversité a été calculée en comptant chaque article disponible dans chaque catégorie d'aliments. Elle inclut les différents formats de vente, de marques, de saveurs et de types. Par exemple, toutes les variétés d'un même fruit ou légume ont été comptées séparément.

La longueur d'étalage a été calculée à l'aide d'une méthode fondée sur la longueur des pas $^{43}$. L'évaluateur a marché devant chaque étalage offrant des aliments inclus dans l'outil en comptant ses pas, qui avaient été préalablement calibrés. Afin de mesurer l'accessibilité des aliments pour les acheteurs, des observations ont été menées dans chaque allée, autour des îlots de présentation et à proximité des caisses enregistreuses. Lorsqu'une catégorie d'aliments était disponible à plusieurs emplacements dans un magasin, les mesures pour tous les emplacements ont été additionnées pour obtenir la longueur d'étalage totale pour cette catégorie d'aliments. La profondeur et la hauteur des étalages n'ont pas été mesurées ni comptabilisées. La longueur d'étalage totale mesurée en additionnant les longueurs d'étalage de tous les groupes d'aliments a permis d'estimer la taille du magasin.

La qualité des produits a été évaluée sur une échelle de trois points, de -1 à 1 . Elle a été vérifiée séparément pour les fruits et les légumes et a été basée sur l'évaluation de leur fraîcheur par l'évaluateur, en fonction 
de l'apparence, de l'odeur et du degré de maturité. Les critères complets d'évaluation de la fraîcheur sont fournis dans le guide d'utilisation de la MEAC-S (disponible auprès des auteurs sur demande, en français uniquement).

2. Le prix des aliments a été évalué selon le prix par portion pour les FL, le prix pour $100 \mathrm{~g}$ de croustilles et de plats congelés et le prix pour 2 litres de boissons gazeuses. Les prix promotionnels n'ont pas été pris en considération.

Le prix par portion pour les fruits et les légumes a été calculé en utilisant, respectivement, le prix moyen pour une portion de pomme, de banane, de fraise et d'orange et le prix moyen pour une portion de tomate, de carotte, de laitue et de concombre. Le Guide alimentaire canadien a servi de référence pour la taille des portions. Lorsque plus d'une variété de ces fruits ou légumes était disponible (par exemple, 17 variétés de pommes), le prix régulier le plus bas a été sélectionné.

Les prix pour $100 \mathrm{~g}$ de croustilles et de plat congelé et pour 2 litres de boissons gazeuses ont été observés pour le produit dont le prix était le plus bas dans chaque magasin, généralement le produit de marque maison. Les évaluateurs ont également enregistré les prix des produits de référence, de marque connue, qui étaient disponibles dans chaque magasin pendant les essais pilotes. Le produit de référence pour les croustilles était le sac de 180 g de croustilles classiques de Lay's et le produit de référence pour les plats congelés était la lasagne de 286 g de Stouffer. La bouteille de Coke de 2 litres était le produit de référence pour les boissons gazeuses.

3. Des indicateurs de proéminence ont été conçus pour décrire l'exposition simultanée à des produits alimentaires sains et non sains. Ceux-ci incluent le " ratio de diversité » et le " ratio de longueur d'étalage » des FL par rapport aux PAUT. Le ratio de diversité a été calculé en divisant le nombre de produits disponibles dans la catégorie FL par le nombre de PAUT disponibles. Le ratio de la longueur d'étalage a été obtenu en divisant la longueur totale d'étalage des FL par la longueur totale d'étalage des PAUT.

4. La promotion des denrées alimentaires a été calculée en utilisant deux indicateurs : le nombre de présentoirs et le positionnement stratégique en magasin des FL, des croustilles, des boissons gazeuses et des confiseries. Le nombre de présentoirs désigne le nombre de fois où les produits alimentaires ont été trouvés en dehors de leur point de vente principal dans le magasin (par exemple, les croustilles sont disponibles dans de nombreux autres endroits que dans l'allée des croustilles à l'intérieur d'un magasin). Les positions stratégiques en magasin sont les extrémités des allées, les zones proches des caisses enregistreuses et les présentoirs de produits prêts à manger. Les évaluateurs ont noté le nombre de ces positions qui étaient occupées par des FL, des croustilles, des boissons gazeuses et des confiseries.

Nous avons mené une étude pilote dans cinq magasins d'alimentation puis nous avons ajusté la MEAC-S pour faciliter la collecte de données. Le formulaire final de l'outil est illustré à la figure 1. Le guide complet de l'utilisateur est disponible (en français seulement) sur demande auprès des auteurs.

\section{Collecte de données}

Létude a été réalisée dans quatre quartiers du sud-est de Montréal (Canada) possédant un niveau de revenu qualifié de faible à moyen. Ces quartiers sont divisés en huit régions de tri d'acheminement (RTA), correspondant aux trois premiers caractères du code postal. Nous avons évalué tous les supermarchés dans ces RTA.

Nous avons sélectionné les supermarchés en utilisant l'outil de recherche Google Maps. La RTA a été saisie comme terme de recherche principal et les termes " supermarché » ou " épicerie » ont été saisis dans le moteur de recherche local. Nous avons localisé 57 magasins d'alimentation, parmi lesquels 18 étaient des supermarchés. Afin de nous assurer de visiter tous les supermarchés, nous avons systématiquement cherché des magasins d'alimentation en longeant chaque rue principale dans les quatre quartiers. Deux magasins se sont avérés inéligibles pour l'étude, le premier ayant fermé ses portes définitivement et le second étant un dépanneur, et un supermarché a été ajouté à la liste, ce qui nous a donné un total de 17 supermarchés (figure 2 ).

Les données ont été recueillies entre mai et juillet 2015, pour éviter les influences saisonnières sur la disponibilité, le prix et la proéminence des FL.

Nous n'avons pas demandé l'autorisation aux gestionnaires de magasins pour évaluer l'environnement alimentaire dans leur supermarché. Par conséquent, la subtilité était un élément clé de la collecte de données. Le formulaire MEAC-S a été imprimé et plié comme s'il s'agissait d'une liste d'épicerie et l'évaluateur a acheté des produits alimentaires dans chaque magasin visité pour éviter d'attirer une attention indésirable. Aucune intervention des gestionnaires de magasins ni des employés n'a compromis la collecte de données.

\section{Fiabilité inter-évaluateur et test-retest}

En novembre 2015, cinq mois après la première évaluation, deux évaluateurs ont réévalué six magasins pour déterminer la fiabilité inter-évaluateur et test-retest de la MEAC-S.

\section{Analyse statistique}

Nous avons calculé les coefficients de corrélation intraclasse (CCI) avec un modèle d'analyse de la variance à deux facteurs avec effets aléatoires pour obtenir une corrélation absolue et déterminer la fiabilité inter-évaluateur et test-retest de la MEAC-S.

Nous avons calculé la corrélation de Spearman entre les indicateurs de prix et de mise en valeur, ainsi qu'entre la taille du magasin et tous les autres indicateurs de l'environnement alimentaire dans les supermarchés.

Toutes les analyses statistiques ont été effectuées dans SPSS Statistics version 19.0 (IBM Corp., Armonk, New York, É.-U.). Une valeur de $p \leq 0,05$ a été considérée comme statistiquement significative.

\section{Résultats}

Tous les supermarchés inclus dans l'étude étaient des supermarchés appartenant à une chaîne, avec des estimations de ventes annuelles dépassant 150 millions de dollars par chaîne ${ }^{44}$.

Les évaluations ont duré en moyenne 56 minutes (de 32 à 75 minutes). Nous avons évalué la fiabilité inter-évaluateur et test-retest d'après le CCI pour chaque indicateur. Un CCI supérieur à 0,75 indique une excellente corrélation et un CCI entre 
FIGURE 1

Formulaire de mesure de la MEAC-S

Magasin :

Date

d'évaluation :
Adresse :

Durée :

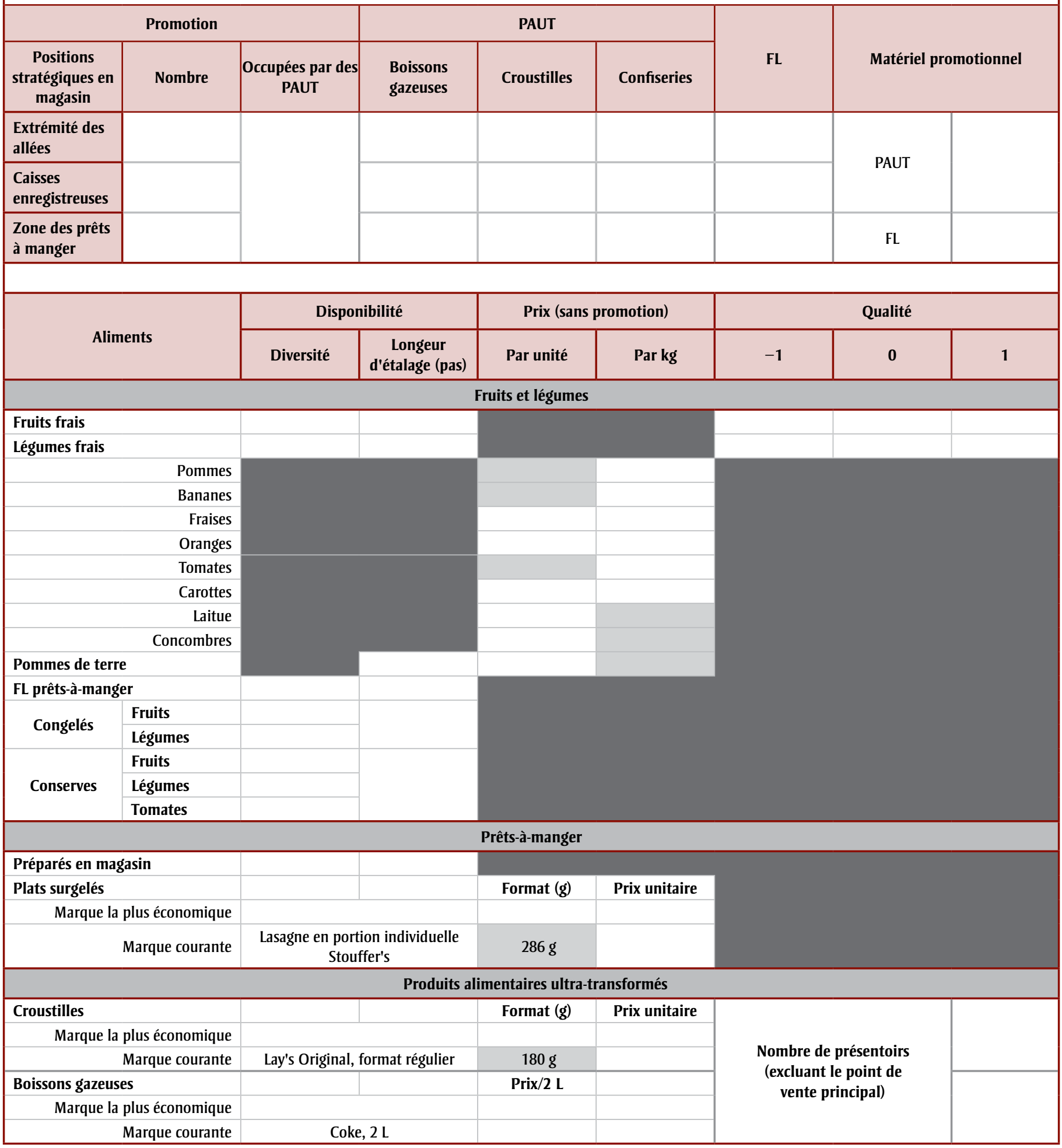

Abréviations : FL, fruits et légumes; MEAC-S, Mesure de l'environnement alimentaire du consommateur dans les supermarchés; PAUT, produits alimentaires ultra-transformés. 


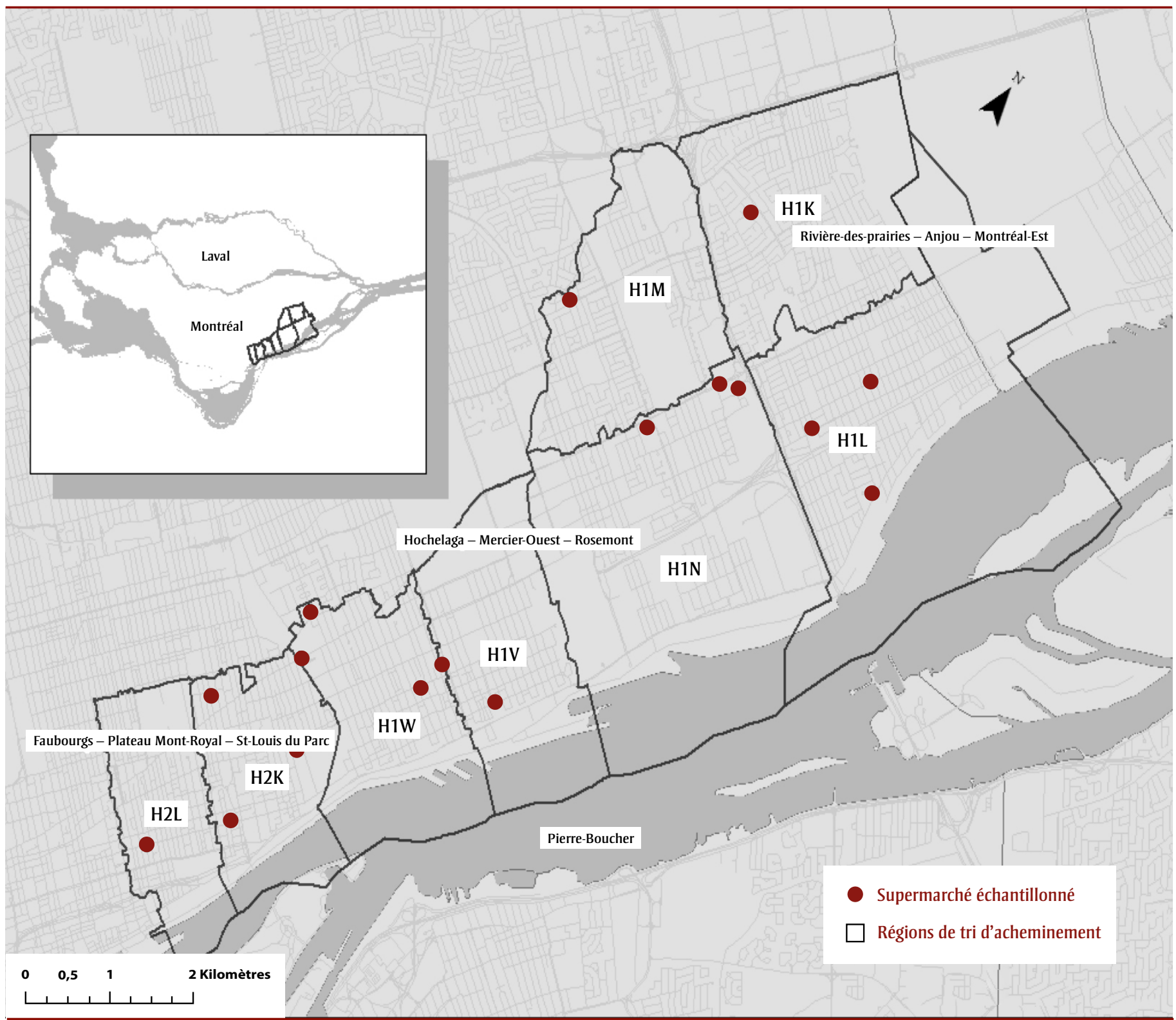

0,40 et 0,75 indique une corrélation moyenne à bonne ${ }^{45}$.

Tous les indicateurs avaient un CCI supérieur à 0,85 pour la fiabilité inter-évaluateur, ce qui suggère un excellent accord entre les évaluateurs. Les CCI pour la fiabilité test-retest étaient plus faibles. Des CCI inférieurs à 0,75 ont été obtenus pour les indicateurs de nombre de présentoirs $(0,43)$ et de positionnement stratégique dans le magasin $(0,53)$ et les coefficients étaient pour la plupart invalides pour les indicateurs de prix en raison d'une variance intragroupe disproportionnée par rapport à la variance intergroupe (tableau 1).

\section{Environnement alimentaire de} consommation

La disponibilité, le prix, la proéminence et la mise en valeur des produits alimentaires par supermarché sont décrits dans le tableau 2.

La disponibilité des produits alimentaires différait considérablement entre les supermarchés, comme en témoigne la variabilité des indicateurs de diversité et de longueur d'étalage. Nous avons calculé des ratios de diversité et de longueur d'étalage pour chacun des magasins. Des ratios supérieurs à 1 indiquent que les FL prédominent par rapport aux PAUT, alors que des ratios inférieurs à 1 indiquent que ce sont les PAUT qui sont davantage présents. Deux supermarchés avaient des ratios de diversité inférieurs à 1,0 et cinq supermarchés avaient des ratios de longueur d'étalage inférieurs à 1,0 .

D'un supermarché à l'autre, le prix par portion de légumes variait du simple au double et le prix par portion de fruits, du simple au triple.

La qualité des FL ne variait pas de manière significative entre les supermarchés, la plupart d'entre eux offrant des FL de la plus haute qualité. 
TABLEAU 1

Fiabilité inter-évaluateurs et test-retest des indicateurs de l'environnement alimentaire de consommation de la MEAC-S

\begin{tabular}{lcc}
\multicolumn{1}{c}{ Indicateurs } & \multicolumn{2}{c}{ CCI } \\
\cline { 2 - 3 } & Inter-évaluateurs & Test-retest \\
\hline Diversité & 0,888 & 0,876 \\
Longueur d'étalage & 0,908 & 0,894 \\
Nombre de présentoirs & 0,951 & 0,431 \\
Qualité & 0,968 & 0,968 \\
Prix & 0,883 & $\mathrm{NV}$ \\
Positionnement stratégique & 0,845 & 0,529 \\
en magasin & & $\mathbf{0 , 6 0 7}$ \\
CCI moyen pour tous les indica- & $\mathbf{0 , 8 9 4}$ & \\
teurs & &
\end{tabular}

Abréviations : $\mathrm{CCl}$, coefficient de corrélation intra-classe; MEAC-S, Mesure de l'environnement alimentaire du consommateu dans les supermarchés; NV, non valide.

Le nombre de présentoirs supplémentaires et de positions stratégiques réservés aux PAUT dépassait largement ceux des FL. Neuf supermarchés n'avaient pas de présentoirs supplémentaires de FL hors de leur section principale de vente, ni de FL disposés dans des positions stratégiques et, parmi les magasins qui en avaient, 6 sur 8 étaient pour des FL en conserve. En comparaison, les PAUT occupaient au minimum 7 présentoirs supplémentaires et 8 positions stratégiques dans tous les magasins.

\section{Prix et mise en valeur des produits alimentaires ultra-transformés}

Les résultats ont montré que le prix des PAUT comme les croustilles et les boissons gazeuses était inversement associé à leur disponibilité, leur proéminence et leur mise en valeur dans les supermarchés (tableau 3). Cette association n'a pas été constatée pour les FL (données non présentées).

\section{Indicateurs de l'environnement alimentaire de consommation et taille du supermarché}

Étant donné que la taille des supermarchés était susceptible d'expliquer certains résultats, nous avons effectué des corrélations de Spearman entre la taille des magasins et les indicateurs de l'environnement alimentaire de consommation, à l'exclusion des mesures de longueur d'étalage (tableau 4).

La taille du magasin a été positivement associée à la diversité, au nombre de présentoirs et au positionnement stratégique des PAUT dans les magasins et inversement associée au prix des boissons gazeuses. La taille du magasin a été associée avec la diversité et le nombre de présentoirs de FL également, mais il convient de noter que des présentoirs supplémentaires pour les FL étaient présents dans seulement 8 supermarchés sur 17 et que la plupart d'entre eux étaient pour les FL en conserve (et non frais).

\section{Analyse}

Le premier objectif de cette étude était de développer un outil pour évaluer l'environnement alimentaire de consommation dans les supermarchés de la province de Québec. La MEAC-S a été utilisée pour mesurer la présence d'aliments sous consommés ou surconsommés au Québec, en utilisant huit indicateurs qui reflètent l'environnement alimentaire des consommateurs dans les commerces.

Les données globales suggèrent une variabilité entre les supermarchés, en particulier en ce qui concerne les mesures de longueur d'étalage et le prix des FL. Le prix des FL variait plus que du simple au double entre les supermarchés. Cela peut entraîner une différence de plus de 30 \$ par semaine pour une famille de quatre personnes selon leur choix de supermarché, ce qui constitue un montant considérable pour les familles à faible revenu résidant dans les quartiers inclus dans l'enquête.

Plusieurs études ont suggéré que le statut socioéconomique du quartier (SSÉQ) est associé à la disponibilité des FL et des grignotines dans les magasins d'alimentation ${ }^{46,47}$, ce qui jouerait un rôle de médiateur dans la relation entre le SSÉQ et la qualité du régime alimentaire ${ }^{33}$. Cependant, les données disponibles sur le SSÉQ dans notre zone d'étude ne correspondent pas à notre répartition géographique, limitant notre capacité à analyser l'environnement alimentaire de consommation dans les différentes RTA en fonction de leur SSÉQ. Les études futures devraient envisager d'utiliser des limites géographiques permettant une intégration adéquate de l'information sur le SSÉQ.

Nos résultats ont également montré que, dans cet échantillon, près de $30 \%$ des 17 supermarchés avaient un ratio de longueur d'étalage inférieur à 1,0 , ce qui indique une prédominance des PAUT dans ces magasins. De plus, le nombre limité de PAUT inclus dans la MEAC-S est susceptible d'aboutir à une sous-évaluation de ce pourcentage.

En outre, nos données suggèrent que les commerces les plus vastes ont un nombre de présentoirs de PAUT plus élevé et que cette catégorie d'aliments occupe davantage de positions stratégiques en magasin que dans les commerces de dimensions plus réduites, une relation qui n'est pas observée pour les FL. Ce résultat correspond aux résultats de recherche précédents montrant que la mise en valeur des aliments malsains semble être liée à la taille des commerces, alors que les FL sont moins présents et sont positionnés dans des endroits moins stratégiques, quelle que soit la taille des commerces ${ }^{40}$. Une étude menée à Montréal par Blanchard a également suggéré que l'espace d'étalage réservé aux grignotines est plus extensible que l'espace d'étalage des $\mathrm{FL}^{46}$.

La plupart des études sur l'environnement alimentaire communautaire classent les supermarchés comme des commerces sains $^{48}$. Bien qu'il ait été démontré qu'ils ont une plus grande disponibilité d'aliments sains à des prix inférieurs aux autres types de commerces ${ }^{33,49}$, ils offrent également plus de PAUT à des prix plus faibles $^{19,50}$ et leur offre alimentaire est très variable d'un supermarché à l'autre ${ }^{20,29}$. Cette étude confirme ces résultats et tend à montrer que les supermarchés ne peuvent pas être considérés uniformément comme des commerces sains. Plusieurs chercheurs exhortent leurs collègues à explorer davantage l'environnement alimentaire de consommation et à réviser leur catégorisation 
TABLEAU 2

Analyse descriptive de l'environnement alimentaire de consommation dans les supermarchés de quatre quartiers de Montréal, Canada

\begin{tabular}{|c|c|c|c|}
\hline \multirow{2}{*}{\multicolumn{2}{|c|}{$\begin{array}{l}\text { Indicateurs de l'environnement } \\
\text { alimentaire de consommation }\end{array}$}} & \multicolumn{2}{|c|}{ Supermarchés $(\mathbf{N}=17)$} \\
\hline & & Moyenne & (min à $\max )$ \\
\hline \multirow{7}{*}{ 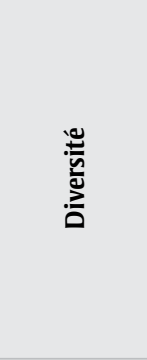 } & FL frais & 221,3 & (149 à 319) \\
\hline & Total (FL) & 518,6 & (361 à 757) \\
\hline & Plats prêts à manger & 79,3 & (0 à 187) \\
\hline & Plats surgelés & 134,5 & (41 à 209) \\
\hline & Croustilles & 235,7 & (123 à 338) \\
\hline & Boissons gazeuses & 41,7 & (27 à 50) \\
\hline & Ratio (FL/PAUT) & 1,28 & $(0,89$ à 1,48$)$ \\
\hline \multirow{8}{*}{ 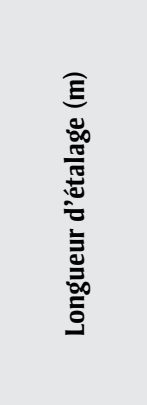 } & FL frais & 89,1 & $(18,2$ à 166,4$)$ \\
\hline & Total (FL) & 123,0 & (34,8 à 223,4) \\
\hline & Plats prêts à manger & 11,2 & $(0,0$ à 29,7$)$ \\
\hline & Plats surgelés & 46,4 & $(18,6$ à 91,5$)$ \\
\hline & Croustilles & 39,6 & $(14,2$ à 82,0$)$ \\
\hline & Boissons gazeuses & 26,8 & $(9,5$ à 70,9$)$ \\
\hline & Ratio (FL/PAUT) & 1,2 & $(0,64$ à 2,34$)$ \\
\hline & Longueur d'étalage totale mesurée & 247,0 & $(88,4$ à 455,3$)$ \\
\hline \multirow{2}{*}{ 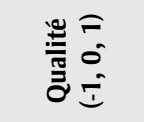 } & $\mathrm{F}$ & 0,8 & $(-0,5$ à 1,0$)$ \\
\hline & $\mathrm{L}$ & 0,9 & $(0,0$ à 1,0$)$ \\
\hline \multirow{8}{*}{ 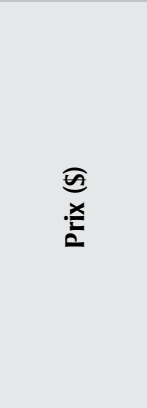 } & F (par portion) & 0,70 & $(0,43$ à 1,22$)$ \\
\hline & $\mathrm{L}$ (par portion) & 0,33 & $(0,22$ à 0,53$)$ \\
\hline & Plats surgelés, MM (par 100 g) & 0,72 & $(0,47$ à 0,93$)$ \\
\hline & Lasagne Stouffer's (par $100 \mathrm{~g}$ ) & 1,41 & $(1,39$ à 1,57$)$ \\
\hline & Croustilles, MM (par $100 \mathrm{~g}$ ) & 0,99 & $(0,74$ à 1,25$)$ \\
\hline & Lay's (par $100 \mathrm{~g}$ ) & 1,63 & $(1,23$ à 1,99$)$ \\
\hline & Boissons gazeuses, MM (par 2 litres) & 1,32 & $(1,00$ à 1,99$)$ \\
\hline & Coke (par 2 litres) & 2,38 & $(1,67$ à 2,79$)$ \\
\hline \multirow{3}{*}{ 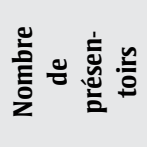 } & $\mathrm{FL}$ & 0,7 & (0 à 3) \\
\hline & Croustilles & 9,9 & (4 à 18) \\
\hline & Boissons gazeuses & 5,5 & (2 à 10) \\
\hline \multirow{4}{*}{ 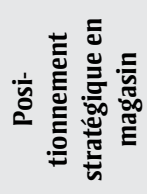 } & $\mathrm{FL}$ & 0,5 & (0 à 2) \\
\hline & Croustilles & 6,9 & (3 à 13) \\
\hline & Boissons gazeuses & 5,7 & (2 à 9) \\
\hline & Confiseries & 10,7 & (3 à 17) \\
\hline
\end{tabular}

Abréviations : F, fruits; FL, fruits et légumes; L, légumes; MM, marque maison; PAUT, produit alimentaire ultra-transformé.

Remarques : Les positions stratégiques en magasin sont l'extrémité des allées, les zones proches des caisses enregistreuses et les étalages de produits prêts-à-manger préparés à l'intérieur des magasins.

La longueur d'étalage totale mesurée est utilisée comme indicateur de la taille du magasin.

des commerces comme étant sains ou malsains ${ }^{23,29,32,51}$.

Contrairement à la disponibilité et au prix, la qualité des fruits et des légumes ne variait pas d'un magasin à l'autre. Ceci est alimentaire entre différents types de magasins. Il convient également de mentionner que dans la plupart des supermarchés que nous avons visités, la section des fruits et des légumes était située à l'entrée du magasin. Ces choix ne sont probablement pas arbitraires et peuvent refléter les pratiques de commercialisation des propriétaires de magasins. En effet, une étude américaine rapporte que les consommateurs qui choisissent d'acheter des aliments perçus comme sains, comme les FL frais, sont plus susceptibles de choisir d'acheter des produits de haute densité énergétique et des produits ultra-transformés plus tard lors de la même visite en magasin ${ }^{52}$.

Cette étude a également révélé une relation inverse entre le prix et la mise en valeur et la proéminence des PAUT, mais pas des FL. Le prix, la proéminence et la mise en valeur sont connus pour avoir une influence majeure sur les comportements d'achats alimentaires. La recherche en marketing suggère que l'augmentation de l'espace d'étalage, la réduction du prix et la disposition des produits aux extrémités des allées ou près des caisses enregistreuses ont toutes pour effet d'augmenter les ventes des produits ainsi mis en valeur ${ }^{14,37-40}$

Il est important de noter que les PAUT étaient promus de cette manière dans tous les magasins inclus dans l'étude, ce qui n'était pas le cas pour les FL. Non seulement le positionnement stratégique d'articles malsains dans les magasins augmente-t-il la vente de ces articles en pourcentage du chiffre d'affaires total, mais il réduit également l'achat de $\mathrm{FL}^{40}$. Compte tenu de l'influence de la proéminence et de la mise en valeur sur les ventes, une stratégie prometteuse pour améliorer l'environnement alimentaire de consommation sans compromettre la rentabilité des commerces pourrait être d'encourager leurs gestionnaires, par des incitatifs financiers ou des règlements, à appliquer également ce mélange de techniques de marketing aux $\mathrm{FL}^{37,39}$.

\section{Points forts et limites}

Cette étude a plusieurs points forts, mais également plusieurs limites. La fiabilité inter-évaluateur de la MEAC-S a été validée avec des CCI satisfaisants pour tous les indicateurs, ce qui suggère un excellent accord entre les évaluateurs. Les CCI pour la fiabilité test-retest étaient légèrement moins satisfaisants. La production de FL 
TABLEAU 3

Coefficients de corrélation de Spearman pour les indicateurs de promotion et de proéminence et le prix des produits alimentaires ultra-transformés

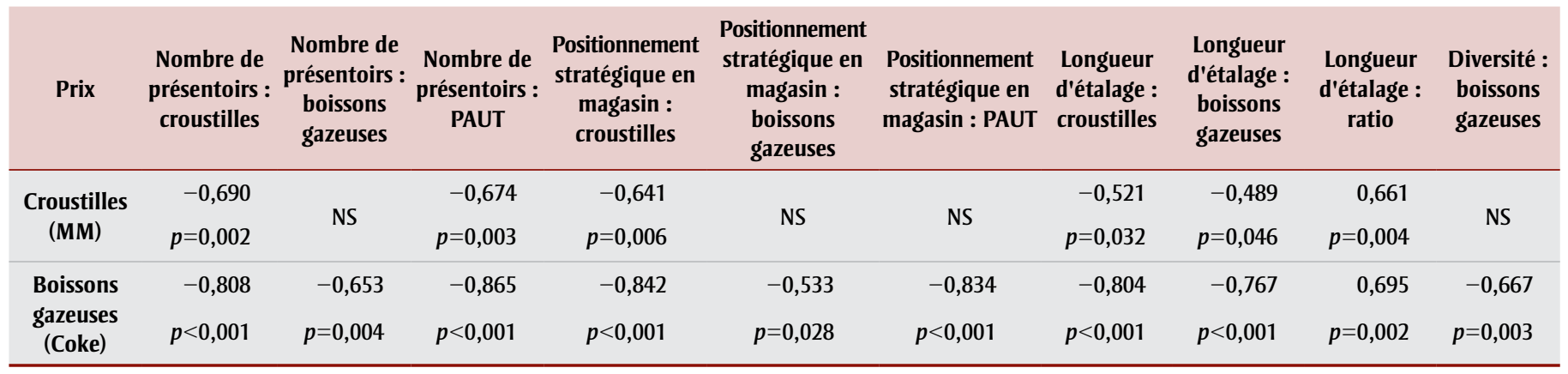

Abréviations : FL, fruits et légumes; MM, marque maison; NS, non significatif; PAUT, produit alimentaire ultra-transformé.

Remarque : Longueur d'étalage : ratio de la longueur d'étalage des FL divisée par la longueur d'étalage des PAUT.

étant étroitement liée au climat et à la température, les variations saisonnières ont une influence sur la disponibilité et le prix des fruits et des légumes en magasin, ce qui pourrait expliquer ces CCI inférieurs. En outre, le nombre de présentoirs et le positionnement des produits alimentaires en magasin peuvent ne pas être constants dans le temps, ce qui pourrait être associé aux particularités ou aux festivités saisonnières. Pour limiter l'influence de la saisonnalité sur les CCI, la fiabilité test-retest de la MEAC-S devrait être réévaluée en utilisant un délai plus court. Il convient également de prendre certaines précautions lors de l'utilisation de la MEAC-S pour évaluer la qualité de l'environnement alimentaire au fil du temps ou lors de la comparaison des magasins ou des quartiers entre eux. Pour maximiser la comparabilité, l'évaluation devrait être effectuée au cours de la même saison.

Les indicateurs et les mesures utilisés constituent un autre point fort de cette étude. L'outil MEAC-S comprend toutes les variétés de chacune des catégories d'aliments étudiées. Bien que l'inclusion d'une aussi grande diversité d'aliments puisse prendre beaucoup de temps, elle pourrait permettre une classification plus sensible des magasins d'alimentation en ce qui a trait à la disponibilité des FL et des produits alimentaires. (Par exemple, une étude réalisée à Montréal n'a pas permis de détecter de différence entre les magasins selon leur SSÉQ en utilisant la liste de contrôle NEMS-S pour les FL frais, mais a détecté une différence significative lorsqu'une liste de contrôle maison de 137 FL frais était utilisée ${ }^{46}$.)

En outre, la MEAC-S intègre des mesures de proéminence et de mise en valeur des aliments, comme le nombre de présentoirs supplémentaires et le positionnement stratégique des aliments dans les magasins, qui se sont révélés étroitement liés aux comportements d'achat ${ }^{37,39}$. À notre connaissance, il s'agit de la première étude à intégrer ces deux mesures dans une enquête sur les magasins d'alimentation. La MEAC-S combine également des indicateurs absolus et relatifs, ce qui illustre mieux l'exposition simultanée des consommateurs aux produits alimentaires sains et malsains $^{29,48,49}$.

Enfin, contrairement à la méthode proposée par d'autres outils d'évaluation, les indicateurs de la MEAC-S n’ont pas été regroupés pour obtenir un score de qualité globale par supermarché. Les résultats des différents indicateurs ne convergeaient pas et étaient parfois opposés en ce qui a trait à la qualité de l'environnement alimentaire dans les supermarchés (p. ex. corrélation positive entre le prix par portion de légume et la proéminence des FL). L'agrégation de ces résultats contrastés ne donnerait pas une image complète et précise de la situation et pourrait sous-estimer l'importance de la relation d'un ou de plusieurs indicateurs avec des comportements d'achat ou des résultats de santé des consommateurs. En outre, les indicateurs de l'environnement alimentaire de consommation peuvent ne pas être liés de la même manière aux habitudes alimentaires ou avoir le même poids pour chaque segment de population ${ }^{51}$. La MEAC-S, en générant des données pour de multiples indicateurs, permet d'effectuer une analyse pour chaque composante de l'environnement alimentaire de consommation en lien avec des résultats alimentaires ou sanitaires.

La principale limite de la MEAC-S est l'inclusion d'un nombre limité de produits alimentaires à évaluer. Cette limitation était délibérée afin de s'assurer du caractère pratique et simple à utiliser de l'outil, en

TABLEAU 4

Coefficients de corrélation de Spearman pour la taille du magasin et les indicateurs de l'environnement alimentaire de consommation dans les supermarchés de quatre quartiers de Montréal, Canada

\begin{tabular}{|c|c|c|c|c|c|c|c|c|c|c|}
\hline & \multicolumn{3}{|c|}{ Diversité } & \multicolumn{3}{|c|}{ Nombre de présentoirs } & \multirow{2}{*}{$\begin{array}{c}\text { Prix } \\
\text { Boissons } \\
\text { gazeuses } \\
\text { (Coke) }\end{array}$} & \multicolumn{3}{|c|}{ Positionnement stratégique en magasin } \\
\hline & FL & Croustilles & PAUT & FL & Croustilles & PAUT & & Croustilles & Confiseries & PAUT \\
\hline Taille du & 0,527 & 0,784 & 0,655 & 0,577 & 0,821 & 0,772 & $-0,695$ & 0,735 & 0,583 & 0,760 \\
\hline magasin & $p=0,030$ & $p<0,001$ & $p=0,004$ & $p=0,015$ & $p<0,001$ & $p<0,001$ & $p=0,002$ & $p=0,001$ & $p=0,014$ & $p<0,001$ \\
\hline
\end{tabular}

Abréviations : FL, fruits et légumes; PAUT, produits alimentaires ultra-transformés. 
particulier pour les praticiens de santé publique, qui manquent souvent de temps et de ressources humaines, principalement en raison de contraintes budgétaires. L'exclusion des PAUT autres que les croustilles, les boissons gazeuses, les plats congelés et les confiseries est susceptible de sous-estimer la proéminence de cette catégorie de produits dans notre environnement alimentaire. De plus, la MEAC-S ne fournit pas d'information concernant les options saines dans ces groupes d'aliments ou d'autres catégories d'aliments disponibles, comme les céréales et les protéines.

Une autre limite importante de cette étude est l'exclusion des magasins d'alimentation autres que les supermarchés. Il y avait une grande variété d'autres types de magasins d'alimentation dans la zone d'étude, comme des petites épiceries et des fruiteries. Au Québec, ces types de magasins représentaient $12,2 \%$ des achats d'aliments en 2013 , tandis que $55,2 \%$ des aliments avaient été achetés en supermarché ${ }^{53}$. Par conséquent, en excluant d'autres types de magasins, il est possible que nous ayons obtenu une représentation erronée de l'environnement alimentaire des résidents de ces quartiers ${ }^{29}$. Cependant, la plupart des consommateurs ont tendance à choisir les supermarchés comme magasins d'alimentation de base et à visiter d'autres types de magasins pour des achats moindres ou complémentaires entre leurs visites régulières aux supermarchés ${ }^{17,33}$. L'inclusion de tous les supermarchés dans les quatre quartiers permet donc probablement de couvrir une bonne partie de l'environnement alimentaire auquel la plupart des résidents sont exposés.

Afin de mieux cerner l'accessibilité financière aux produits alimentaires, les prix promotionnels ont été exclus des observations, malgré leur influence connue sur les comportements d'achat ${ }^{54}$. D'autres études pourraient évaluer la fréquence des réductions de prix par catégorie d'aliments en plus du nombre de présentoirs et du positionnement stratégique en magasin. Le petit échantillon de supermarchés et le milieu urbain de niveau socioéconomique faible à moyen choisis pour l'étude constituent également des limites, empêchant une généralisation des résultats aux zones rurales ou à revenu élevé.

\section{Conclusion}

À notre connaissance, la MEAC-S est le premier outil permettant d'évaluer l'environnement alimentaire de consommation en utilisant un ensemble de mesures intégrant non seulement la disponibilité et le prix, mais aussi des indicateurs de proéminence et de mise en valeur. Nos résultats illustrent une mise en valeur et une proéminence des PAUT supérieures à celles des FL dans les quartiers étudiés et soulignent la nécessité de brosser un tableau adéquat de l'environnement alimentaire auquel les consommateurs sont exposés, en évitant une classification dichotomique des magasins en commerces sains ou non sains. La MEAC-S pourrait être utilisée pour caractériser et surveiller de manière fiable l'environnement alimentaire de consommation dans les supermarchés, et pourrait donc fournir les données nécessaires pour guider les interventions et les politiques ciblant l'environnement alimentaire en vue d'améliorer les habitudes alimentaires à l'échelle de la population.

\section{Remerciements}

Ce projet a été soutenu en partie par un programme de bourses d'études supérieures du Canada des Instituts de recherche en santé du Canada (IRSC).

\section{Références}

1. Statistique Canada. Base de données CANSIM : Tableau 105-0507. Indice de masse corporelle (IMC) mesuré chez les adultes, selon le groupe d'âge et le sexe, population à domicile de 18 ans et plus excluant les femmes enceintes, Canada (sauf les territoires) [Internet]. Ottawa (Ont.) : Statistique Canada; 2016. En ligne à : http://www5.statcan .gc.ca/cansim/a26? id $=1050507 \&$ retrLang $=$ fra\&lang $=$ fra

2. Tran BX, Nair AV, Kuhle S, Ohinmaa A, Veugelers PJ. Cost analyses of obesity in Canada: scope, quality, and implications. Cost Eff Resour Alloc [Internet]. 2013;11(1):3. doi: 10.1186 /1478-7547-11-3

3. Blouin C, Hamel D, Vandal N, Jen Y, Lo E, Martel S. Les conséquences économiques associées à l'obésité et l'embonpoint au Québec: les coûts liés à la consommation de médicaments et à l'invalidité - mise à jour 2016. Québec (Québec) : Institut national de santé publique Québec (INSPQ); 2015. (Rapport $\mathrm{n}^{\circ}$ 2035)
4. Consultation OMS/FAO d'experts, éditeur. Régime alimentaire, nutrition et prévention des maladies chroniques : rapport d'une consultation OMS/FAO d'experts. Genève : Organisation mondiale de la santé; 2003. (Série de Rapports techniques $n^{\circ}$ 916)

5. Blanchet C, Rochette L, Plante C, Institut national de santé publique du Québec (INSPQ). La consommation alimentaire et les apports nutritionnels des adultes québécois [Internet]. Montréal : INSPQ; 2009. En ligne à : https:// www.inspq.qc.ca/pdf/publications /931_RapportNutritionAdultes.pdf

6. Moubarac J-C, Batal M. La consommation d'aliments transformés et la qualité de l'alimentation au Québec [Internet]. Montréal (Québec) : Transnut; 2016. En ligne à : http://www.rccq.org /wp-content/uploads/Qu \% C3\% A9bec -MSSS-consommation-daliments-ultra -transform \% C3\% A9s-et-qualit\% C3\% A9 -de-lalimentation_Moubarac-et-Batal -2016.pdf

7. Glanz K, Sallis JF, Saelens BE, Frank LD. Healthy nutrition environments: concepts and measures. Am J Health Promot. 2005;19(5):330-333, ii.

8. Caspi CE, Kawachi I, Subramanian SV, Adamkiewicz G, Sorensen G. The relationship between diet and perceived and objective access to supermarkets among low-income housing residents. Soc Sci Med. 2012;75(7):1254-1262.

9. Izumi BT, Zenk SN, Schulz AJ, Mentz GB, Wilson C. Associations between neighborhood availability and individual consumption of dark-green and orange vegetables among ethnically diverse adults in Detroit. J Am Diet Assoc. 2011;111(2):274-279.

10. Morland K, Wing S, Diez Roux A. The contextual effect of the local food environment on residents' diets: the Atherosclerosis Risk in Communities study. Am J Public Health. 2002; 92(11):1761-1767.

11. Rose D, Richards R. Food store access and household fruit and vegetable use among participants in the US Food Stamp Program. Public Health Nutr. 2004;7(8):1081-1088. 
12. Zenk SN, Lachance LL, Schulz AJ, Mentz G, Kannan S, Ridella W. Neighborhood retail food environment and fruit and vegetable intake in a multiethnic urban population. Am J Health Promot. 2009;23(4):255-264.

13. Aggarwal A, Cook AJ, Jiao J, et al. Access to supermarkets and fruit and vegetable consumption. Am J Public Health. 2014;104(5):917-923.

14. Cheadle A, Psaty BM, Curry S, et al. Community-level comparisons between the grocery store environment and individual dietary practices. Prev Med. 1991;20(2):250-261.

15. Moore LV, Diez Roux AV, Nettleton JA, Jacobs DR. Associations of the local food environment with diet quality-a comparison of assessments based on surveys and geographic information systems: the multi-ethnic study of atherosclerosis. Am J Epidemiol. 2008; 167(8):917-924.

16. Franco M, Diez-Roux AV, Nettleton JA, et al. Availability of healthy foods and dietary patterns: the Multi-Ethnic Study of Atherosclerosis. Am J Clin Nutr. 2009;89(3):897-904.

17. Gustafson A, Christian JW, Lewis S, Moore K, Jilcott S. Food venue choice, consumer food environment, but not food venue availability within daily travel patterns are associated with dietary intake among adults, Lexington Kentucky 2011. Nutr J [Internet]. 2013; 12:17. En ligne à : http://dx.doi.org/10 $.1186 / 1475-2891-12-17$

18. Gustafson A, Lewis S, Perkins S, Wilson C, Buckner E, Vail A. Neighbourhood and consumer food environment is associated with dietary intake among Supplemental Nutrition Assistance Program (SNAP) participants in Fayette County, Kentucky. Public Health Nutr. 2013;16(7): 1229-1237.

19. Rose D, Hutchinson PL, Bodor JN, et al. Neighborhood food environments and body mass index: the importance of in-store contents. Am J Prev Med. 2009;37(3):214-219.
20. Chaix B, Bean K, Daniel $M$, et al. Associations of supermarket characteristics with weight status and body fat: a multilevel analysis of individuals within supermarkets (RECORD Study). PLOS ONE [Internet]. 2012;7(4):e32908. En ligne à : https://doi.org/10.1371 /journal.pone.0032908

21. Morland K, Diez Roux AV, Wing S. Supermarkets, other food stores, and obesity: the Atherosclerosis Risk in Communities study. Am J Prev Med. 2006;30(4):333-339.

22. Spence JC, Cutumisu N, Edwards J, Raine KD, Smoyer-Tomic K. Relation between local food environments and obesity among adults. BMC Public Health [Internet]. 2009;9:192. En ligne à : https://doi.org/10.1186/1471-2458 $-9-192$

23. Le $H$, Engler-Stringer R, Muhajarine N. Walkable home neighbourhood food environment and children's overweight and obesity: proximity, density or price? Can J Public Health. 2016; 107(Suppl 1):eS42-eS47.

24. Minaker LM, Raine KD, Wild TC, Nykiforuk CIJ, Thompson ME, Frank LD. Objective food environments and health outcomes. Am J Prev Med. 2013;45(3):289-296.

25. Powell LM, Auld MC, Chaloupka FJ, O'Malley PM, Johnston LD. Associations between access to food stores and adolescent body mass index. Am J Prev Med. 2007;33(4 Suppl): S301-S307.

26. Moubarac J-C, Batal M, Martins AP, et al. Processed and ultra-processed food products: consumption trends in Canada from 1938 to 2011. Can J Diet Pract Res. 2014;75(1):15-21.

27. Myers CA, Slack T, Martin CK, Broyles ST, Heymsfield SB. Change in obesity prevalence across the United States is influenced by recreational and healthcare contexts, food environments, and hispanic populations. PLOS ONE [Internet]. 2016;11(2):e0148394. En ligne à : https://doi.org/10.1371/journal .pone.0148394
28. Swinburn BA, Sacks G, Hall KD, McPherson K, Finegood DT, Moodie $\mathrm{ML}$, et al. The global obesity pandemic: shaped by global drivers and local environments. Lancet. 2011; 378(9793):804-814.

29. Lucan SC. Concerning limitations of food-environment research: a narrative review and commentary framed around obesity and diet-related diseases in youth. J Acad Nutr Diet. 2015; 115(2):205-212.

30. Minaker LM, Shuh A, Olstad DL, Engler-Stringer R, Black JL, Mah CL. Retail food environments research in Canada: a scoping review. Can J Public Health. 2016;107(Suppl 1):eS4-eS13.

31. Odoms-Young A, Singleton CR, Springfield S, McNabb L, Thompson $\mathrm{T}$. Retail environments as a venue for obesity prevention. Curr Obes Rep. 2016;5(2):184-191.

32. Caspi CE, Sorensen G, Subramanian SV, Kawachi I. The local food environment and diet: a systematic review. Health Place. 2012;18(5):1172-1187.

33. Cannuscio CC, Tappe K, Hillier A, Buttenheim A, Karpyn A, Glanz K. Urban food environments and residents' shopping behaviors. Am J Prev Med. 2013;45(5):606-614.

34. Glanz K, Johnson L, Yaroch AL, Phillips M, Ayala GX, Davis EL. Measures of retail food store environments and sales: review and implications for healthy eating initiatives. J Nutr Educ Behav. 2016;48(4):280-288.e1.

35. Glanz K, Sallis JF, Saelens BE, Frank LD. Nutrition Environment Measures Survey in Stores (NEMS-S): development and evaluation. Am J Prev Med. 2007;32(4):282-289.

36. Carlson A, Lino M, Juan W, Hanson K, Basiotis PP. Thrifty Food Plan, 2006. Washington (DC): US Department of Agriculture, Center for Nutrition Policy and Promotion; 2007. (Rapport no CNPP-19)

37. Curhan RC. The effects of merchandising and temporary promotional activities on the sales of fresh fruits and vegetables in supermarkets. J Mark Res. 1974;11(3):286-294. 
38. Wilkinson JB, Mason JB, Paksoy $\mathrm{CH}$. Assessing the impact of short-term supermarket strategy variables. J Mark Res. 1982;19(1):72-86.

39. Foster GD, Karpyn A, Wojtanowski AC, Davis E, Weiss S, Brensinger C, et al. Placement and promotion strategies to increase sales of healthier products in supermarkets in low-income, ethnically diverse neighborhoods: a randomized controlled trial. Am J Clin Nutr. 2014;99(6):1359-1368.

40. Kerr J, Sallis JF, Bromby E, Glanz K. Assessing reliability and validity of the GroPromo audit tool for evaluation of grocery store marketing and promotional environments. J Nutr Educ Behav. 2012;44(6):597-603.

41. Monteiro C, Cannon G, Levy RB, Claro RM, Moubarac J-C. The food system. Ultra-processing. The big issue for nutrition, disease, health, well-being. [Commentaire]. World Nutr. 2012; 3(12):527-569.

42. Base de données privée de M.-C. Paquette (Université de Montréal, Montréal [Québec]), accès de septembre 2015 à décembre 2016.

43. Bertrand L, Thérien F, Agence de la santé et des services sociaux de Montréal (Québec), Direction de santé publique. Les disparités dans l'accès à des aliments santé à Montréal : une étude géomatique [Internet]. Montréal (Québec) : Direction de santé publique Montréal, Agence de la santé et des services sociaux de Montréal; 2006. En ligne à : https://publications .santemontreal.qc.ca/uploads/tx _asssmpublications/2-89494-516-7.pdf

44. Paquette M-C, Bergeron P. Cadre de référence des indicateurs d'achats pour caractériser l'alimentation et l'environnement alimentaire au Québec [Internet]. Montréal : Institut national de santé publique du Québec; 2016. En ligne à : https://www.inspq.qc.ca /publications/2180

45. Fleiss JL, Levin B, Paik MC. The measurement of interrater agreement. Dans : Statistical methods for rates and proportions [Internet]. Hoboken (NJ): John Wiley and Sons; 2003. p. 598626. En ligne à : http://onlinelibrary .wiley.com/doi/10.1002/0471445428 .ch18/summary
46. Blanchard L. Disparities in the availability of fruit, vegetables and snack foods by neighborhood socioeconomic status in supermarkets and grocery stores in Montréal, Canada [mémoire de maîtrise]. Copenhagen (Danemark Copenhagen University and University of Sheffield; 2012.

47. Zenk SN, Powell LM, Rimkus L, et al. Relative and absolute availability of healthier food and beverage alternatives across communities in the United States. Am J Public Health. 2014; 104(11):2170-2178.

48. Cobb LK, Appel LJ, Franco M, JonesSmith JC, Nur A, Anderson CAM. The relationship of the local food environment with obesity: a systematic review of methods, study quality, and results. Obes (Silver Spring). 2015;23(7): 1331-1344.

49. Farley TA, Rice J, Bodor JN, Cohen DA, Bluthenthal RN, Rose D. Measuring the food environment: shelf space of fruits, vegetables, and snack foods in stores. J Urban Health. 2009;86(5): 672-682.

50. Miller C, Bodor JN, Rose D. Measuring the food environment: a systematic technique for characterizing food stores using display counts. J Environ Public Health [Internet]. 2012;2012: e707860. En ligne à : http://dx.doi.org $/ 10.1155 / 2012 / 707860$

51. Gustafson A, Hankins S, Jilcott S. Measures of the consumer food store environment: a systematic review of the evidence 2000-2011. J Community Health. 2012;37(4):897-911.

52. Hui SK, Bradlow ET, Fader PS. Testing behavioral hypotheses using an integrated model of grocery store shopping path and purchase behavior. J Consum Res. 2009;36(3):478-493.

53. Labrecque J, Dupuis R, Doyon $M$, Dufour J-C. Approvisionnement du marché alimentaire québécois : des stratégies gagnantes [Internet]. Montréal : CIRANO; 2016 (Rapport no 2016RP06). En ligne à : https://www.cirano .qc.ca/files/publications/2016RP-06 .pdf
54. Nakamura R, Suhrcke M, Jebb SA, Pechey R, Almiron-Roig E, Marteau TM. Price promotions on healthier compared with less healthy foods: a hierarchical regression analysis of the impact on sales and social patterning of responses to promotions in Great Britain. Am J Clin Nutr. 2015; ajcn.094227. 